\title{
IASI-NG development status
}

\section{Bertrand Calvel, Francois Faure, Antoine Penquer}

Bertrand Calvel, Francois Faure, Antoine Penquer, "IASI-NG development status," Proc. SPIE 11852, International Conference on Space Optics - ICSO 2020, 118523Y (11 June 2021); doi: 10.1117/12.2599631 SPIE. Event: International Conference on Space Optics - ICSO 2021, 2021, Online 


\section{International Conference on Space Optics-ICSO 2020}

Virtual Conference

30 March-2 April 2021

Edited by Bruno Cugny, Zoran Sodnik, and Nikos Karafolas
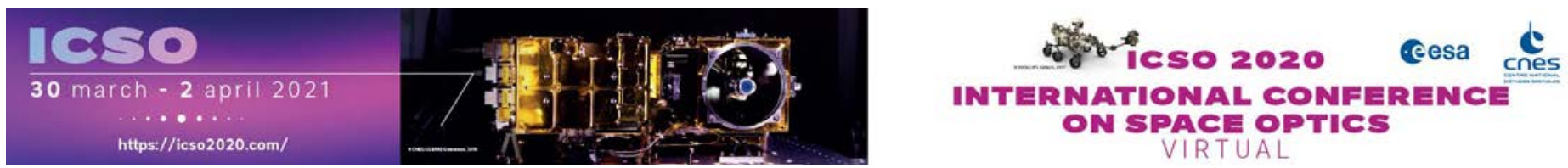

\section{IASI-NG development status}

\section{Cesa isopmeatians ecnes}

International Conference on Space Optics - ICSO 2020, edited by Bruno Cugny, Zoran Sodnik, Nikos Karafolas, Proc. of SPIE Vol. 11852, 118523Y · @ 2021 ESA and CNES

CCC code: $0277-786 \mathrm{X} / 21 / \$ 21 \cdot$ doi: $10.1117 / 12.2599631$ 


\title{
IASI-NG development status
}

\author{
Bertrand Calvel $^{* a}$, Antoine Penquer ${ }^{* * b}$ \\ a Airbus Defence and Space SAS, France, 31, rue des Cosmonautes 31402 Toulouse Cedex, France; \\ ${ }^{\mathrm{b}} \mathrm{CNES}, 18$, Avenue Edouard Belin 31400 Toulouse, France
}

\begin{abstract}
The Infrared Atmospheric Sounding Interferometer New Generation (IASI-NG) is a key payload element of the second generation of European meteorological polar-orbit satellites (METOP SG) dedicated to operational meteorology, oceanography, atmospheric chemistry, and climate monitoring.

It will continue and improve the IASI mission in the next decades (2020-2040) in the field of operational meteorology, climate monitoring, and characterization of atmospheric composition related to climate, atmospheric chemistry and environment. The performance objective is mainly a spectral resolution and a radiometric error divided by two compared with the IASI first generation ones.

The measurement technique is based on wide field Fourier Transform Spectrometer (operating in the 3.5 - $15.5 \mu \mathrm{m}$ spectral range) based on an innovative Mertz compensated interferometer to manage the so-called self-apodization effect and the associated spectral resolution degradation.

The alignment, integration and test phase has started on the flight model. We present here the results obtained on the subsystem environment qualification and performance tests and the status of the complete flight model integration. This includes the performance vacuum tests on the complete Focal Plane and Cooler Assembly and the performance vacuum tests on the assembly made of the aligned flight Interferometer with its Laser Metrology. The obtained results show the complete end to end processing of the acquired interferograms taking into account all metrology data.
\end{abstract}

CNES is in charge of the overall IASI-NG mission. The instrument is manufactured by Airbus Defence \& Space.

Keywords: Fourier Transform Interferometer, IASI, KBr, infrared, METOP-SG

\section{INTRODUCTION}

IASI-NG (Infrared Atmospheric Sounding Interferometer New Generation) is the follow on mission of IASI. It shall provide operational meteorology data such as temperature and humidity atmospheric profiles and also monitor other gases like ozone, methane or carbon monoxide on a global scale.

The IASI-NG instrument performs spectral measurement in the infrared between $3.6 \mu \mathrm{m}$ and $15.5 \mu \mathrm{m}$ using Fourier Transform interferometry. Compared to IASI, the new generation instrument shall improve the radiometric signal to noise ratio and the spectral resolution by a factor two. Table 1 gives the main instrument requirements:

Table 1. IASI-NG main requirements

\begin{tabular}{|l|l|l|}
\hline Parameter & Requirement & Value \\
\hline Geometry & sounding point size & $\approx 12 \mathrm{~km}$ \\
& spatial sampling & $\approx 25 \mathrm{~km}$ \\
& geolocation error & $0.5 \mathrm{~km}$ \\
\hline Radiometry & radiometric calibration error & $0.25 \mathrm{~K} @ 280 \mathrm{~K}$ \\
& NedT@280K & 0.1 to $0.4 \mathrm{~K}$ within the spectrum \\
\hline Spectral & continuous spectral covering & From $3.6 \mu \mathrm{m}$ to $15.5 \mu \mathrm{m}$ \\
& spectral resolution & $0.25 \mathrm{~cm}^{-1}$ \\
& spectral sampling & $0.12 \mathrm{~cm}^{-1}$ \\
& spectral calibration error & $510^{-7}$ \\
\hline
\end{tabular}

\footnotetext{
*bertrand.calvel@airbus.com; phone (33) 562196383

**antoine.penquer@cnes.fr; phone (33) 561281657
} 
Due to volume and mass constraints it was not possible to improve IASI radiometric performances by increasing the pupil size. The chosen solution is to increase the Field Of View (FOV) size and thus the integration time. The resulting FOV is in the order of $100 \times 100 \mathrm{~km}^{2}$. The spectral resolution and sampling are divided by two by doubling the Optical Path Difference (OPD) of the interferometer to about $8 \mathrm{~cm}$ in total $( \pm 4 \mathrm{~cm})$.

Unfortunately Michelson interferometers present a so called self-apodisation effect which reduces the signal level when one increases the FOV and the OPD range. Contrarily to the previous IASI instrument, this effect would reduce the signal to null before reaching the extreme OPD with the IASI-NG parameters. It is thus mandatory to compensate this effect to get a sufficient signal level. The chosen solution principle was proposed by Mertz and consists in putting in the optical path a refractive material which thickness changes with the OPD. This is made in IASI-NG by having two opposite prisms moving synchronously with the OPD change. A patented mechanism concept allows performing simultaneously the OPD scan and the compensation using a single actuator. In order to achieve good performances in the Infrared domain, the selected material for the prisms and separation optics is $\mathrm{KBr}$ providing a flat response until $15,5 \mu \mathrm{m}$.

\section{INSTRUMENT DESCRIPTION SUMMARY}

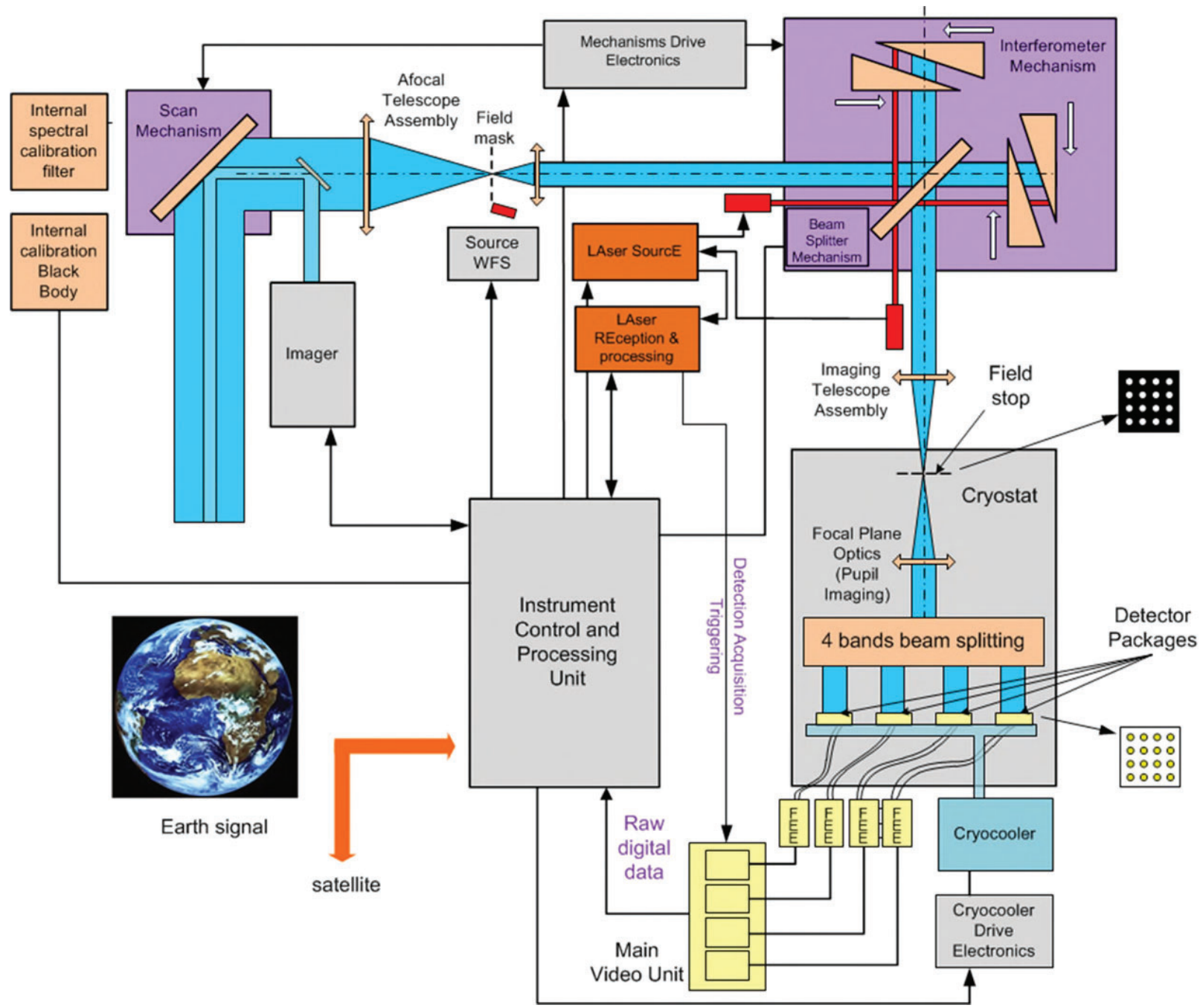

Figure 1. block diagramme of the IASI-NG instrument 
A detailed instrument description and principle explanation has been given in ref [1]. We then only recall here the main functions.

The beam enters the instrument via a two axes pointing mirror used to select the Earth views and to compensate the satellite velocity. It then passes through an afocal telescope used to reduce the pupil size before entering the interferometer. At interferometer output, an imaging telescope focuses the beam on the field mask located at the entrance of the cryostat containing the four detection arrays for the four spectral bands.

A stabilized laser metrology beam source (LASE) divided into five sub-beams is also sent inside the interferometer and acquired and processed by the LARE electronics. This electronics is in charge of triggering the detection using the central metrology beam to provide constant OPD sampling and to record interferometer elements displacements for the ground post-processing of the interferograms using the other four lateral beams.

\section{INTERFEROMETER DEVELOPMENT}

\subsection{Alignment}

The interferometer design and alignment has been entirely performed by Airbus Defence \& Space in Toulouse. The interferometer must be aligned such that:

- The optical elements are put at their right position with the proper orientation

- The mechanical elements (pivots) are such that the optical path scan is made without tilt of the wavefront larger than $10 \mu \mathrm{rad}$ in order to keep a good modulation efficiency

The alignment has been performed using a 3D measuring machine equipped with an optical non-contact sensor. This was necessary because the $\mathrm{KBr}$ prisms and plates are too soft to allow mechanical contact. Before alignment, the optics are also characterized in their mounts equipped with reference sapphire balls using the same machine.

The obtained results measured by looking at the wavefront variations over the full range show that the various elements have been successfully aligned. The corresponding accuracy is within a few microns for a global interferometer dimensions in the $0.5 \mathrm{~m}$ range.

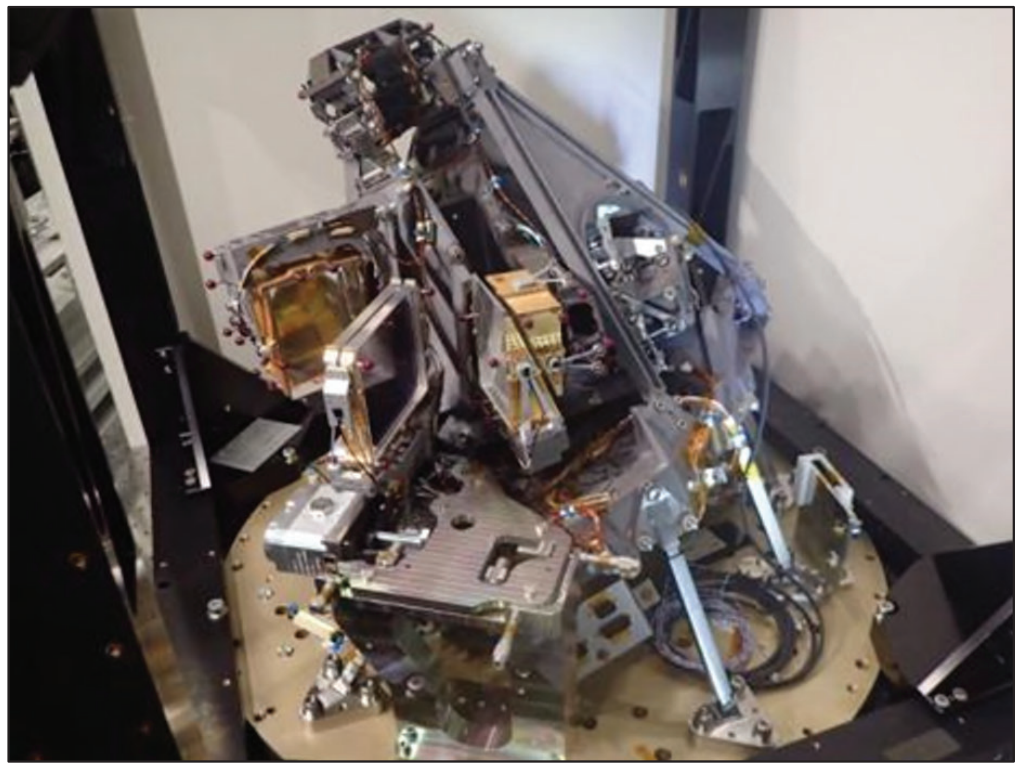

Figure 2. The interferometer with its optics and flex pivots aligned with micrometer accuracy

As the metrology beams do not follow exactly the science beam path in the interferometer, it is necessary to take into account the actual wavefront maps of the various elements of the optical train. These elements are thus independently 
measured before integration. This is also useful to verify that they are not submitted to stress during mounting and to predict the system final performance. At interferometer level, we are able to measure the global wavefront of each interferometer arm as well as the differential wavefront at output. These predictions and measurement are made for several path values to take into account the relative displacements of the prisms during the optical path scan.

These measurements have been performed on both EQM and FM1 models and the correlation between individual measurements and aligned interferometer is very good as shown here under.

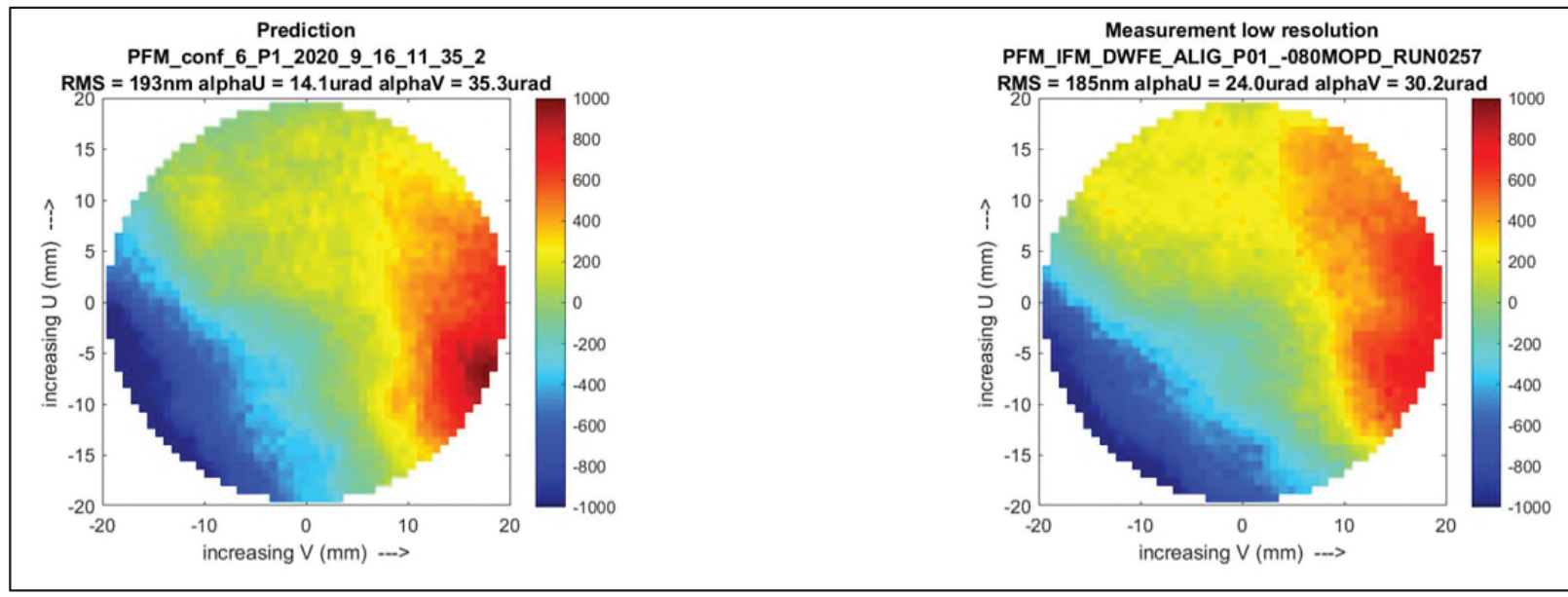

Figure 3. The measured interferometer wavefront has been found in very good accordance with the predicted one

After final alignment a test has been performed by coupling the mechanism to its drive electronics. This test has demonstrated that we are indeed able to cover the required OPD range with speed stability compatible with the performance of the metrology. We are thus able to sample the interferogram with the required accuracy, without generating visible self-induced microvibrations.

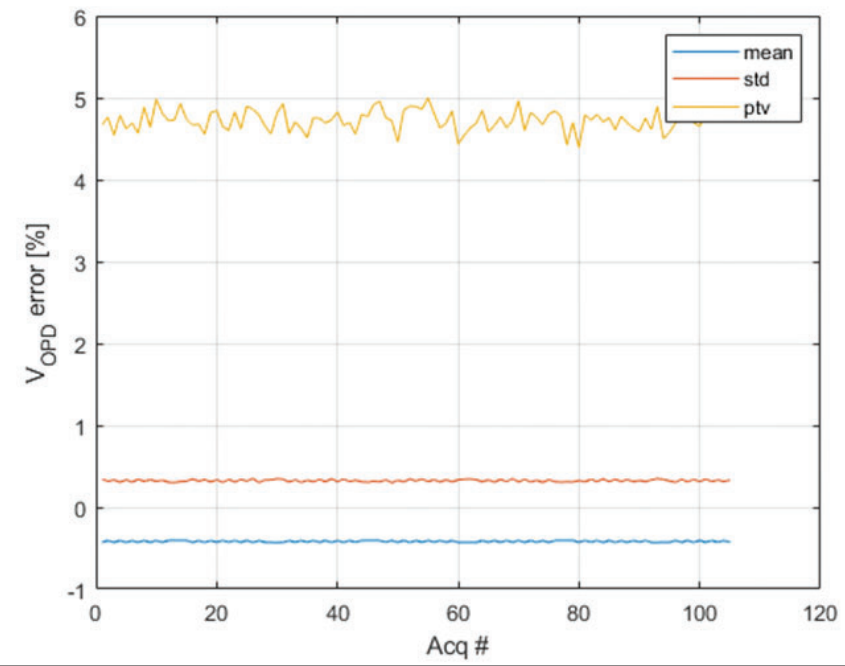

Figure 4. The optical path difference scan is made with a speed stability in the few percent range, thus allowing a good sampling performance for the interferograms. 


\subsection{Environment Qualification}

The interferometer is working in an environment regulated stable within a few tenths of Kelvin in operation. However its stability has been qualified with respect to non-operational thermal temperatures between $-10^{\circ} \mathrm{C}$ and $+45^{\circ} \mathrm{C}$.

For mechanical environment the interferometer has been submitted to quasistatic levels between 12 and $17 \mathrm{~g}$ depending on axis, sinus and random, compatible with METOP-SG launch. Together with the interferometer, the Beam Splitter angle adjustment mechanism which allows in orbit fine alignment tuning has also been qualified.

The integrity of the hardware was checked after these tests by looking at the variations in WFE and tilt variation along the optical path scan range. The results as shown in the figure below were well within the requirements.

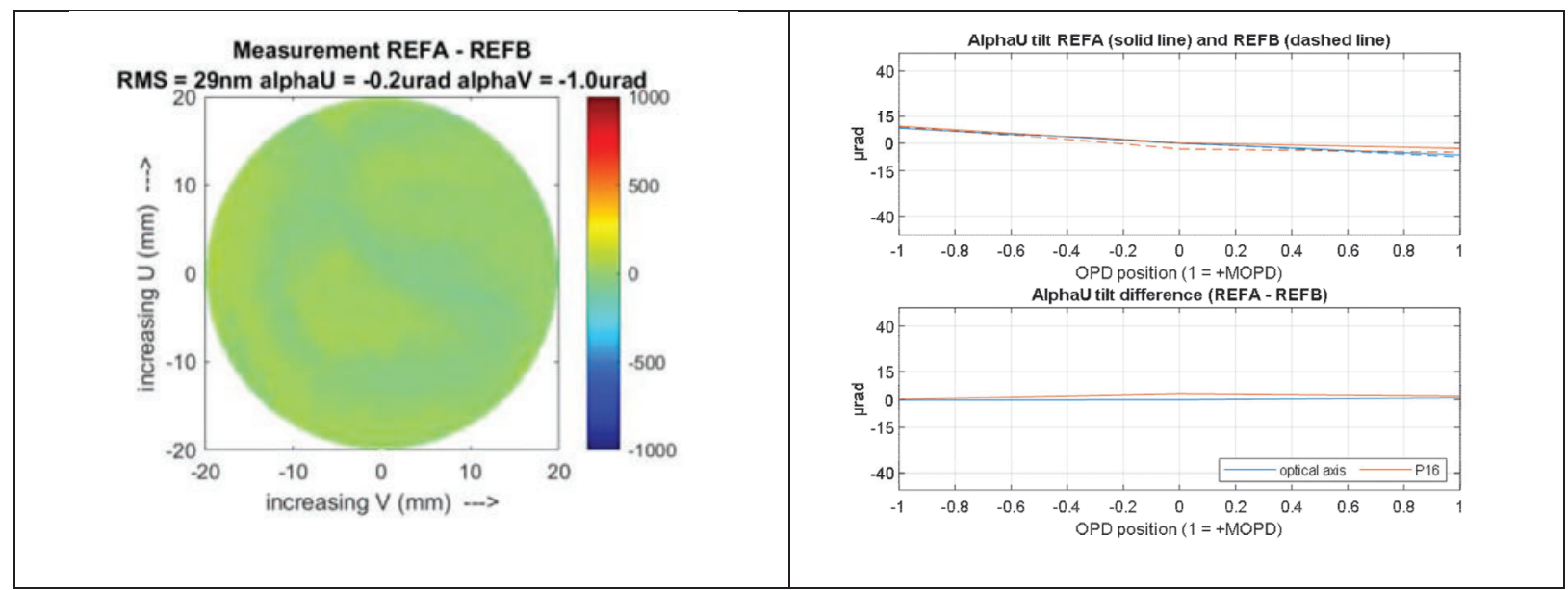

Figure 5. WFE variation in the order of $30 \mathrm{~nm}$ rms and mechanism tilt variations below $7 \mu$ rad were measured after interferometer environment tests.

\section{FOCAL PLANE AND CRYO-COOLER ASSEMBLY PERFORMANCES}

The FPCA has already been successfully qualified with respect to thermal and mechanical environment as reported in ref [1]. Since, the EQM focal plane was fully aligned and tested in the EQM cryostat and its performances validated. The EQM assembly was also used for an extensive EMC test campaign with detectors operated at $80 \mathrm{~K}$ with the full detection chain, showing no spikes nor susceptibility of the complete chain. The flight model focal plane has now been aligned and a set of performance tests has been held in November 2020. For these tests the cryostat is evacuated and the detection is cooled down to its operating temperature about $80 \mathrm{~K}$ using the flight cryocoolers. An optical test setup working at ambient conditions is installed in front of the system. This setup provides black bodies sources and moving targets. This allows performing knife edge measurements as well as crosstalk characterization by illuminating one sounder pixel at a time. First order radiometric evaluations were also performed.

The measured performances were within the requirements with only exception of minor non-conformances for the cross talk in some channels, still compatible with mission requirements. The FM focal plane in its cryostat and cold optics is now ready to be integrated on the instrument baseplate.. 


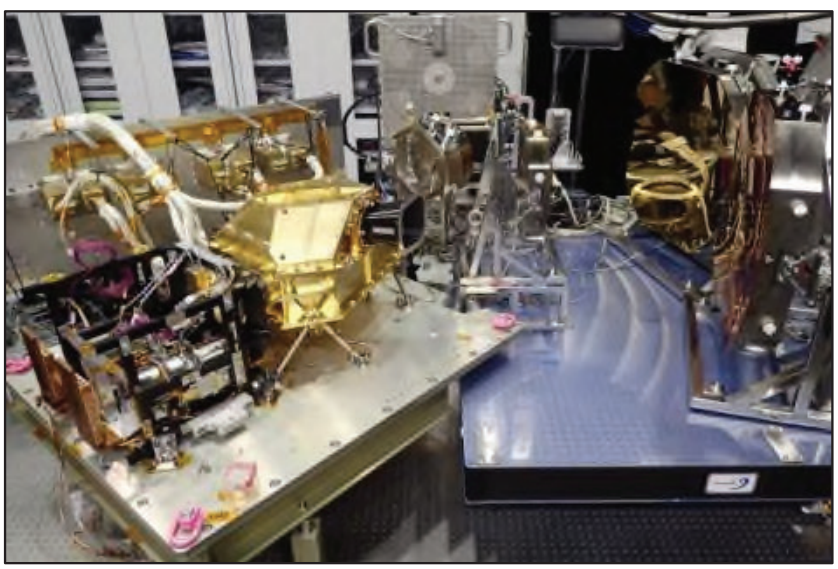

Figure 6. Flight model focal plane assembly in cold vacuum placed in front of the test optical system.

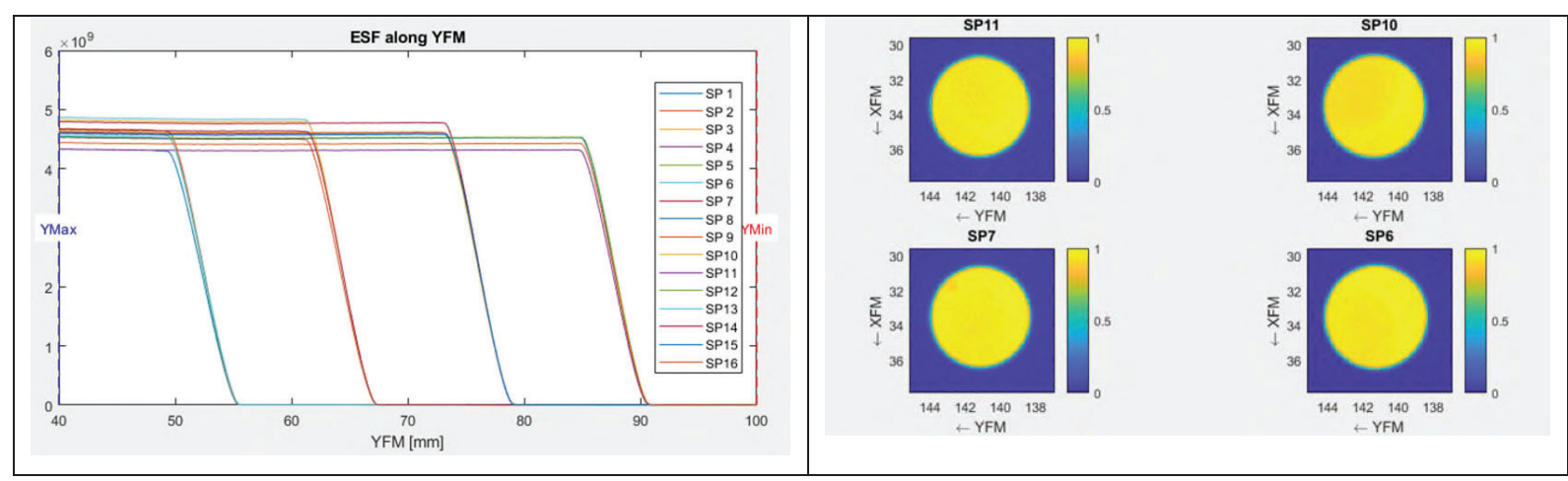

Figure 7. Examples of Knife Edge and Point Spread functions during focal plane cold vacuum test. Although the test setup is at ambient conditions, the quality of the obtained data is very good.

\section{TELESCOPES}

IASI NG comprises two telescopes. The Afocal Telescope Assembly is used to reduce the pupil size from 92 to $40 \mathrm{~mm}$ in order to have a reasonably sized interferometer. The Imaging Telescope Assembly then focuses the beams on the field stop located inside the cryostat.

Both telescopes are three mirror telescopes with off axis aspheric as well as free form mirrors. They have been polished by REOSC. The telescope structure as well as the mirrors are made of Silicon Carbide to take advantage of its specific stiffness and stability. Their alignment has been performed using code V simulations and an excel based solver. The alignment constraints include not only the wavefront error but also the Line Of Sight and the position of the entrance and exit pupils. One has to be sure that these parameters are properly located within the mechanical reference frame of the telescopes so that the alignment at instrument level is compatible with the existing limited adjustment ranges.

The wavefront requirements are in the micron range as these optics are used for wavelengths above $3.6 \mu \mathrm{m}$ and for an instrument which is not an imager. The pupil position has been obtained within $0.5 \mathrm{~mm}$ accuracy and the LOS within 0.5 mrad, thus well-fitting instrument needs. 


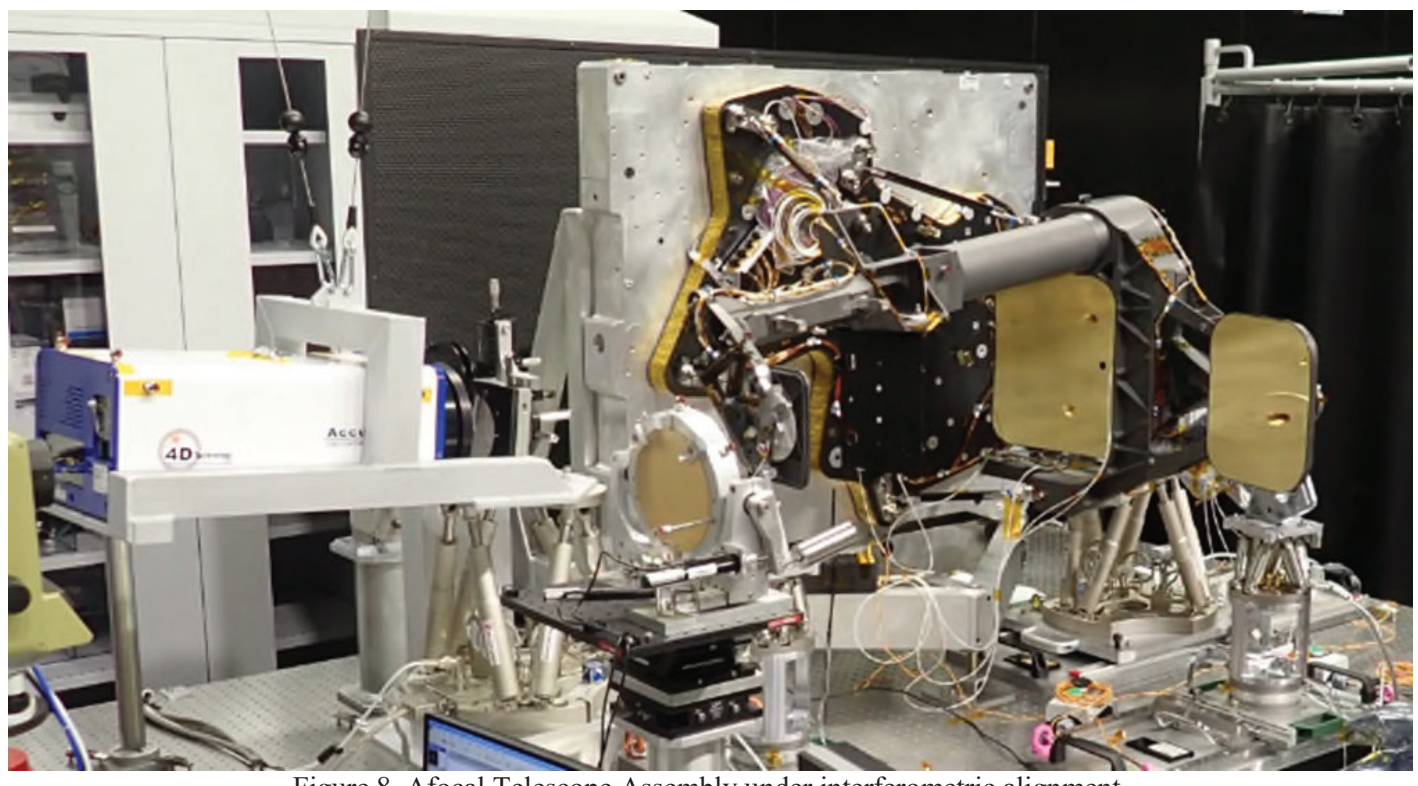

Figure 8. Afocal Telescope Assembly under interferometric alignment

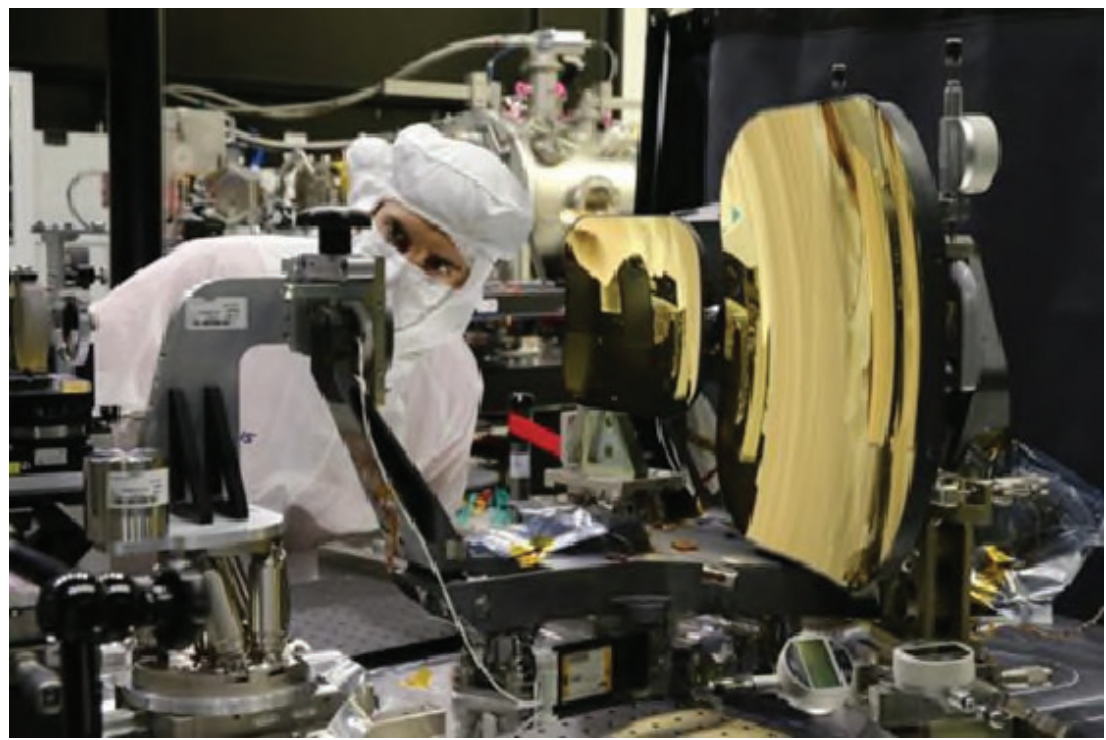

Figure 9. Imaging Telescope during alignment

\section{SYSTEM PERFORMANCE VACUUM TEST}

\subsection{Overview}

The most important test so far for which concluded the development phase of the instrument is the Interferometer Assembly performance test in thermal vacuum which has been performed end of 2020.

Several sources are used in vacuum for this test, namely a warm and a cold black body, a gas cell with a calibrated gas mixture, a laser source and the Fabry Perot filter used for in orbit spectral calibration monitoring. 
The detection is limited to a single sounder pixel to allow for use of simplified telescope optics in this test and the waveband is the 3.6 to $4.4 \mu \mathrm{m}$ band which is the most demanding in term of optical quality.

The assembly under test is the flight model interferometer actuated with its Mechanism Drive Electronics (EM MDE from Syderal). The laser metrology is also included with a stabilized Laser Source (EM LASE form Kongsberg) and the Laser Receiving Electronics (EM LARE from EREMS) in charge of metrology processing. This processing is used to sample in real time the interferogram with equal path differences and to monitor the interferometer additional perturbations for on ground post processing. The tests used an EM detector, EM Front End Electronics (FEE from CRISA) and the PFM Video Chain Electronics (MVU PFM from Thales Alenia Space Toulouse).

\subsection{Test Results}

This test has allowed demonstrating the following functions and performances:

- Proper interferogram acquisition with equal increments of optical path (constant OPD sampling)

- Proper synchronization between the science interferograms and the metrology data

- $\quad$ End to end processing of interferograms to recover spectra

The procedure for in flight alignment using the motorized beam splitter adjustment mechanism has been validated as well as the good performance of interferometer active thermal control in the presence of external perturbations.

Here below are shown a few samples of data acquired during this system test that have allowed verifying the end to end functions and performances of the core of the instrument. The FM1 interferometer is now ready for integration on the instrument.

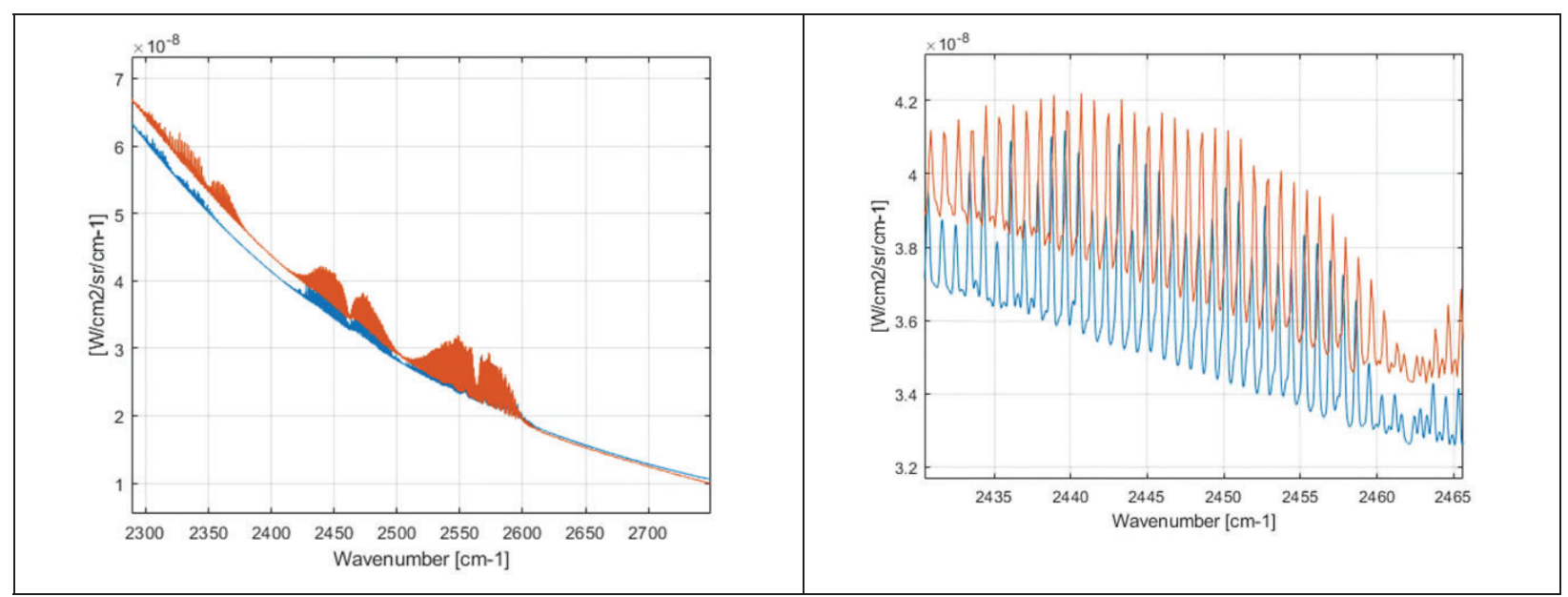

Figure 10. (blue) model and (orange) test data from gas cell acquisition showing the good correlation between results and predictions, the spectral shift should disappear when the on-going spectral calibration processing is included. 


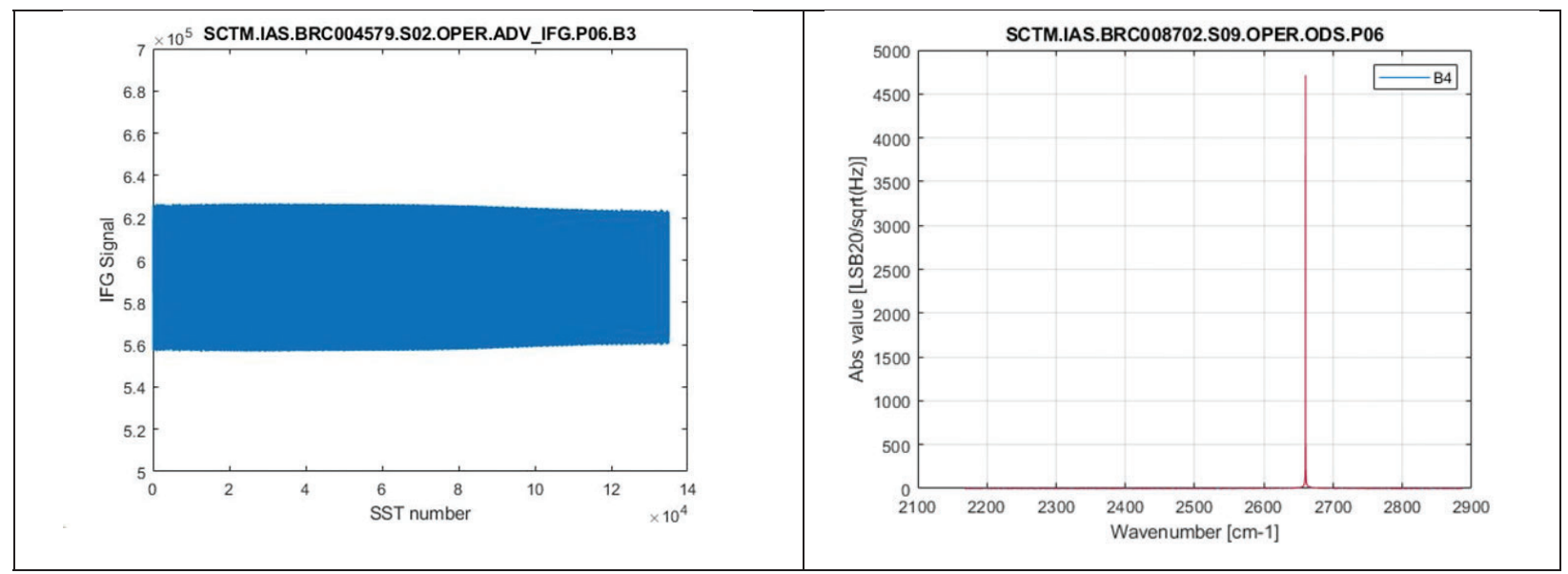

Figure 11. interferogram and corresponding spectrum for the laser source.

\section{FUTURE ACTIVITIES}

The Critical Design Review of the instrument has been held end 2019.

As shown above the sub-systems of the flight model have been integrated. The integration and alignment of the flight instrument is on-going with the Optical Head on one side and the coupling of the Electronics Module on the other side.

The EMC qualification will take place on the EM instrument in April. On the flight model, the integration will be followed by the mechanical environment acceptance tests and the final full instrument performance test under vacuum.

The delivery of the flight model is expected during the last quarter of 2021, it is then mounted onto the satellite platform.

\section{REFERENCES}

[1] Calvel, B., Bernard, F., “IASI-NG development status,” Proc. ICSO Conference, Oct. 2018. 